\title{
Autologous Serum Skin Test in Chronic Idiopathic Urticaria
}

\author{
Pokhrel $K^{1}$, Subedi $S^{2}$, Acharya $S^{3}$
}

\begin{abstract}
Background: Urticaria is a short-lived swelling of skin and mucosa due to plasma leakage by immune and non immune mediated activation and released of mediators from mast cell and basophil. Autoimmune urticaria tends to have a high itch and wheal score than other type of urticaria. Its diagnosis is practically relied upon clinical suspicion and autologous serum skin test. Autologous serum skin test (ASST) is the simple and cost effective test to differentiate autoimmune urticaria from the bulk of chronic urticaria patients. Objective: To compare the features of chronic urticaria in patients having positive versus negative autologous serum skin test (ASST). Materials and methods: Cross-sectional hospital based study was conducted among 90 chronic urticaria patients (CIU) attending the outpatient dermatology department of Nepalgunj Medical College, Nepalgunj, during one year period. The study was conducted after ethical approval from the institutional committee. The patients were diagnosed on the basis of the appearance of continuous or recurrent hives with or without angioedema for more than 6 weeks. Patients who suffered from either acute urticaria or urticarial vasculitis or physical urticaria or other systemic diseases known to cause urticaria were excluded. Standard tools and techniques were used to prepare autologous serum and injection of the serum and interpretation of the result. The test result was interpreted as positive and negative autologous serum skin test. Results: ASST was positive in $42 \%$ of the patients and negative in $58 \%$ of the patients. The ASST-positive patients had a higher mean urticaria activity score and median duration of wheals in comparison with the ASST-negative patients. Wheals lasted for significantly longer duration in patients with positive ASST. Patients with positive ASST had more frequent attacks which was statistically significant compared to the ASST-negative group. The mean urticaria activity score was significantly higher in the ASST-positive patients than that in the ASST-negative patients. Conclusions: . Autologous serum skin test may be a useful screening test for autoimmune urticaria and may be used as a simple and cost-effective test for the classification of chronic urticaria.
\end{abstract}

Key words: Autologous serum skin test (ASST), chronic idiopathic urticaria (CIU), Immunosupressive drugs, urticaria activity score (UAS)

\section{INTRODUCTION}

Chronic idiopathic urticaria (CIU) is one of the commonest skin disorder characterized by the recurrent eruption of shortlived(lasting less than 24 hours) wheals accompanied by redness and itching occurring daily or almost daily for at least 6 weeks and where a predominant physical cause has been excluded $^{1}$. CIU is extremely disabling in its severe form and can be difficult to treat. An affected patient often goes from one dermatologist to another for a cure. Mast cell degranulation is the major pathogenesis of CIU. Until recently, all remaining cases were assigned to the category of CIU since no definite cause of CU was known in those cases. This issue was partially resolved in 1993 when Hide et $\mathrm{al}^{2}$ demonstrated that autoantibodies against the high-affinity IgE receptor, $F_{c} \varepsilon R, \alpha$ cause histamine release in a subset of patients with CU.

\footnotetext{
1. Dr. Kumar Pokhrel

2. Dr. S. Subedi

3. Dr. S. Acharya
}

\author{
Address for correspondence: \\ Dr. Kumar Pokhrel \\ Department of Dermatology \\ Nepalgunj Medical College Teaching Hospital \\ Nepalgunj, Banke, Nepal \\ E-mail: dr@kumarpokhrel.com.np
}

Further subsequent reports showed that $27-61 \%$ of $\mathrm{CU}$ patients, depending on the method of antibody detection, had these circulating antibodies in their blood of CU patients $s^{3,45,6}$. The simplest screening method to identify this group of patients having what was termed chronic autoimmune urticaria (CAU), was found to be the autologous serum skin test (ASST) ${ }^{7}$. Diagnosing these patients as autoimmune urticaria becomes important because uses of systemic immunosuppressive drugs are not justified in chronic idiopathic urticaria unless it is diagnosed as autoimmune in origin. Various methods have been used to categorize chronic autoimmune urticaria. The basophil histamine release assay is currently the gold standard for detecting these functional auto antibodies in the serum of patients with chronic urticaria. However, this bioassay is difficult to standardize because it requires fresh basophils from healthy donors, is timeconsuming and it remains confined to research centers. Western analysis, enzyme-linked immunosorbent assays (ELISA) and flow cytometry may be useful for screening in the future and they need validation.

Autologous serum skin test (ASST) is the simplest and the best in vivo clinical test for the detection of basophil histaminereleasing activity. The ASST has a sensitivity of $70 \%$ and a specificity of $80 \%$ for in vitro basophil histamine release when the serum response is at least $1.5 \mathrm{~mm}$ greater than the control 
saline skin test at $30 \mathrm{~min}^{7}$. Intradermal injection of autologous serum in these patients elicited an immediate-type wheal and flare response indicating the presence of a circulating histamine-releasing factor that is functional auto antibodies that activate mast cells and basophils by cross linking the highaffinity IgE receptor $\left(F_{c} \varepsilon R, \alpha\right)$. About $50 \%$ patients of chronic urticaria have functional auto antibodies to $F_{c} \varepsilon R, \alpha$ and $9 \%$ to the IgE antibody itself ${ }^{8,9}$. Autoimmune urticaria is thus diagnosed in ASST-positive chronic urticaria patients who exhibit functional autoantibodies against IgE and/or its highaffinity receptor FceRI. It is often clinically difficult to distinguish chronic urticaria from autoimmune urticaria. Its diagnosis is practically relied upon clinical suspicion and autologous serum skin test (ASST) which facilitates for assaying functional histamine release from basophils or mast cells. Patient with CAU tends to have a greater number of wheals with a wider distribution, more severe pruritus and more frequent systemic symptoms ${ }^{10}$. Chronic autoimmune urticaria may be associated with other autoimmune diseases like thyroid disease, vitiligo, diabetes mellitus, pernicious anemia and rheumatoid arthritis ${ }^{11}$. It can reasonably be used as a predictive clinical test to diagnose clinically suspected cases of autoimmune urticaria or at least a subgroup of chronic urticaria patients who are more likely to have an endogenous or autoimmune cause for their disease ${ }^{12}$. For the benefit to patients and dermatologists regarding long-term management in terms of need of using supra pharmacological doses of antihistamines or immunomodulators, the study was conducted with an objective to compare the features of chronic urticaria in patients having positive versus negative autologous serum skin test (ASST).

\section{MATERIALS AND METHODS}

A cross-sectional hospital based study was conducted among $90 \mathrm{CIU}$ patients attending the outpatient dermatology department of Nepalgunj Medical College, Nepalgunj from March 2013 to February 2014. Approval of the study was obtained from the institutional committee. Patients were enrolled in the study for ASST after taking informed written consent. CIU patients were diagnosed on the basis of the appearance of continuous or recurrent hives with or without angioedema for more than 6 weeks ${ }^{1}$. The study patients were of either sex and more than 15 years of age and who were off antihistamines for 3 days, 7 days for long-acting antihistamines and 2 weeks for doxepin and corticosteroids or immunosuppressive agents for 6 weeks to 3 months as per the procedure $^{7,12}$.

Patients having acute urticaria, urticarial vasculitis, physical urticaria (diagnosed on the basis of history/provocation tests) or other systemic diseases known to cause urticaria were excluded. Pregnant and lactating mothers were also excluded. Routine investigations like complete blood count, random blood sugar, urine and stool examination were done to exclude chronic urticaria cases that were not idiopathic. All the patients were investigated for thyroid function test (TFT). The ASST test was not performed over the areas involved by wheals in the last 24 hours. The ASST was considered positive when the average diameter of the autologous serum-induced wheal was $>1.5 \mathrm{~mm}$ of the saline-induced wheal. Symptoms and signs were graded on the basis of the modified urticaria activity score $[\text { Table I] }]^{10}$. Age, gender, age of onset, duration, frequency of episodes, number and distribution of individual urticarial which was recorded at the time of clinical examination were analyzed in relation to the ASST results. The Chi Square, Fischer's exact tests, Mann-Whitney test were applied. P-value less than 0.05 , calculated at the $5 \%$ level ( $95 \%$ confidence limit), was considered to be statistically significant.

Pruritus severity score
Absent $=0$
Present but not disturbing $=1$
Disturbing but not hampering daytime activity or sleep = 2
Hampering daytime activity or sleep $=3$
Wheel score (average no. of wheals in $24 \mathrm{~h}$ )
Less than 10 wheals = 1
$10-50$ wheals $=2$
$>50$ wheals $=3$
Involving almost the whole body $=4$

\section{Table I: Urticaria activity score}

A numerical value is assigned to signs/symptoms(ignoring the wheals size). Urticaria activity score $=$ Pruritus severity score + Wheel score

\section{Preparation of Autologous serum and controls}

Two milliliters of patient's venous blood was collected in a sterile glass tube and allowed to clot for $30 \mathrm{~min}$ at room temperature. The serum was then separated by centrifugation at $2000 \mathrm{rpm}$ for $15 \mathrm{~min}$ and used immediately for ASST and sterile physiological saline $(0.9 \%)$ for negative control was used $^{3,13}$.

\section{Procedure and Interpretation}

Approximately $0.05 \mathrm{ml}$ (equivalent to 2 units on insulin syringe that has $1 \mathrm{ml}$ marked as 40 units) each of autologous serum, and sterile physiological saline was injected by a separate syringe intradermally over volar aspect of the left forearm. Autologous serum was injected proximally and normal saline distally keeping a gap of at least $5 \mathrm{~cm}$ between the two injection sites. After 30 minute the wheal formed at each injection site is measured at two perpendicular diameters $\left(d_{1}\right.$ and $\left.d_{2}\right)$ and the average of the two is calculated $d^{10,13,14,15}$.

Positive ASST was the one with serum-induced wheal which had a diameter (average of $d_{1}$ and $d_{2}$ ) of $\geq 1.5 \mathrm{~mm}$ as compared to the saline-induced wheal at $30 \mathrm{~min}$. Using this criterion, the sensitivity and specificity of the ASST for detecting autoantibodies is 70 and $80 \%$, respectively, and false positive results in healthy subjects and controls are minimal. 


\section{RESULTS}

Ninety patients participated in the study. About $31 \%$ were in age group of $26-35$ years followed by $46-55$ years $(27.8 \%)$. Mean age of the participants was $31.59 \pm 13.15$ years. Most of the patients were females $63(70 \%)$. TFT was abnormal among 6 (6.7\%) patients. Autologus serum skin test was positive among $38(42.2 \%)$ patients (Table II). With respect to gender, age group and TFT, there was no statistical difference ( $p>0.05)$ between the groups of patients with ASST positive and ASST negative. Gender and age had no effect on the status of ASST. The cases in ASST positive and ASST negative group were comparable with regard to status of TFT (Table III).

Patients with positive ASST had more frequent attacks which was statistically significant as compared to the ASST-negative group $(p<0.05)$. Urticaria activity score (UAS) of $\geq 5$ was observed in 35 ASST-positive and in 19 ASST-negative patients. UAS was $<5$ in 3 ASST-positive and 33 ASST-negative patients. Urticaria activity score was higher in ASST- positive patients than ASST-negative patients which was statistically highly significant $(p<0.001)$. Patients with higher frequency of attacks and activity score could be important features among patients with ASST-positive. The mean urticaria activity score (6.28 \pm 1.7) was also higher in the ASST-positive patients than that (5.28 \pm 1.7$)$ in the ASST-negative patients which was statistically highly significant $(p<0.001)$. Wheals lasted for significantly longer duration in patients with positive ASST, the median duration being three hours for ASST-positive as compared to one hour in ASST-negative individuals $(p<0.001)$.

Median duration of the disease was 10 months and 13 months in ASST positive and ASST-negative groups, respectively but was statistically insignificant. Duration of disease might not have affect on ASST result (Table IV).

\begin{tabular}{|c|c|c|}
\hline Characteristics & No. of patients & Percentage \\
\hline \multicolumn{3}{|l|}{ Gender } \\
\hline Male & 27 & 30.0 \\
\hline Female & 63 & 70.0 \\
\hline \multicolumn{3}{|l|}{ Age Group } \\
\hline $15-25$ yrs. & 16 & 17.8 \\
\hline $26-35$ yrs. & 28 & 31.1 \\
\hline $36-45$ yrs. & 16 & 17.8 \\
\hline $46-55$ yrs. & 25 & 27.8 \\
\hline$\geq 56$ yrs. & 5 & 5.6 \\
\hline \multicolumn{3}{|l|}{ TFT } \\
\hline Abnormal & 6 & 6.7 \\
\hline Normal & 84 & 93.3 \\
\hline \multicolumn{3}{|c|}{ Autologus serum skin test } \\
\hline Positive & 38 & 42.2 \\
\hline Negative & 52 & 57.8 \\
\hline Mean age (years) & \begin{tabular}{|c|}
$31.59 \pm 13.15$ \\
Min. 15, Max. 61
\end{tabular} & \\
\hline
\end{tabular}

\begin{tabular}{|c|c|c|c|}
\hline \multirow[b]{2}{*}{ Characteristics } & \multicolumn{2}{|c|}{ Autologus serum skin test } & \multirow[b]{2}{*}{ p-value } \\
\hline & $\begin{array}{c}\text { Positive } \\
\text { n1(\%) }\end{array}$ & $\begin{array}{c}\text { Negative } \\
\text { n2(\%) }\end{array}$ & \\
\hline \multicolumn{4}{|l|}{ Gender } \\
\hline Male & $11(40.7)$ & $16(59.3)$ & \multirow{2}{*}{$>0.05$} \\
\hline Female & $27(42.9)$ & $36(57.1)$ & \\
\hline \multicolumn{4}{|l|}{ Age groups (yrs) } \\
\hline $15-25$ & $5(31.3)$ & $11(68.7)$ & \multirow{5}{*}{$>0.05$} \\
\hline $26-35$ & $13(46.4)$ & $15(53.6)$ & \\
\hline $36-45$ & $6(37.5)$ & $10(62.5)$ & \\
\hline $46-55$ & $12(48.0)$ & $13(52.0)$ & \\
\hline$\geq 56$ & $2(40.0)$ & $3(60.0)$ & \\
\hline \multicolumn{4}{|l|}{ TFT } \\
\hline Abnormal & $2(33.3)$ & $4(66.7)$ & \multirow{2}{*}{$>0.05$} \\
\hline Normal & 36 (42.9) & $48(57.1)$ & \\
\hline
\end{tabular}

Table III: Comparison of background characteristics of patients with chronic urticaria according to the status of autologous serum skin test

\begin{tabular}{|c|c|c|c|}
\hline \multirow[b]{2}{*}{ Clinical Features } & \multicolumn{2}{|c|}{ Autologus serum skin test } & \multirow[b]{2}{*}{ p-value } \\
\hline & $\begin{array}{c}\text { Positive } \\
\text { n1(\%) }\end{array}$ & $\begin{array}{c}\text { Negative } \\
\text { n2(\%) }\end{array}$ & \\
\hline \multicolumn{4}{|c|}{ Frequency of attack } \\
\hline Daily & $26(51)$ & $25(49)$ & \multirow{3}{*}{$<0.05$} \\
\hline 1 - 3 per week & $12(34.3)$ & $23(65.7)$ & \\
\hline 1 - 3 per month & $0(0)$ & $4(100)$ & \\
\hline \multicolumn{4}{|c|}{ Urticaria activity score } \\
\hline$\geq 5$ & $35(64.8)$ & $19(35.2)$ & \multirow{2}{*}{$<0.001$} \\
\hline$<5$ & $3(8.3)$ & $33(91.7)$ & \\
\hline $\begin{array}{l}\text { Mean urticaria } \\
\text { activity score }\end{array}$ & $6.28 \pm 1.7$ & $5.28 \pm 1.7$ & $<0.001$ \\
\hline $\begin{array}{l}\text { Duration of } \\
\text { disease (months) } \\
\text { Median }\end{array}$ & 10 & 13 & $>0.05$ \\
\hline $\begin{array}{l}\text { Duration of } \\
\text { wheels (hours) } \\
\text { Median }\end{array}$ & 3 & 1 & $<0.001$ \\
\hline
\end{tabular}

Table IV: Comparison of clinical features of patients with chronic urticaria to the status of autologous serum skin test

Table II: Distribution of patients according to their background characteristics $(n=90)$ 


\section{DISCUSSION}

This study had evaluated patients with chronic idiopathic urticaria (CIU) by autologous serum skin testing to compare the clinical features of patients with positive and negative ASST results. ASST positivity among $42 \%$ of patients with $\mathrm{CIU}$ in this study supported the previous studies showing $27-60 \%$ ASST positivity ${ }^{16-24}$.

The median duration of disease was 10 and 13 months for ASST-positive and negative patients respectively, which was not statistically significant and was comparable to the previous study done by Sabroe et al ${ }^{10}$. Lesions lasted for significantly longer durations in patients with a positive ASST, median duration being three hours when compared to one hours in ASST-negative individuals $(p=0.001)$. Patients with a positive ASST had more frequent attacks, which was statistically significant compared to the ASST-negative group $(p=0.05)$. A study done by Sabroe et al. concluded that patients with autoantibodies in their sera have more severe attacks according to several_parameters including frequency, duration of individual episodes and sites involved ${ }^{10}$. This is in concordance with our study, which noted frequent long-lasting episodes in ASST-positive patients.

However, the overall age and sex profile of our patients in both the groups was statistically comparable to that reported by most workers ${ }^{19-21,23,25}$.

Statistically significant higher UAS of $\geq 5$ in our $65 \%$ ASSTpositive patients as compared with that in 35\% ASST-negative patients which was similar to other studies $(p=0.001)^{10,16-18}$. However, not all studies have shown a significant difference in UAS between the ASST-positive and the ASST- negative groups $^{21,26}$, signifying variable UAS presentation among these patients.

Autoimmune diseases like thyroid disease, vitiligo, diabetes mellitus, pernicious anemia and rheumatoid arthritis were reported more commonly in patients with autoimmune urticaria ${ }^{10,11}$. Our study showed no difference in TFT status in ASST-positive and ASST-negative ones.

The relationship between chronic urticaria and thyroid autoimmunity had been studied by Leznoff et $\mathrm{al}^{27}$ and it has been postulated that thyroid autoimmunity may play a role in the pathogenesis of chronic urticaria and angioedema. However, in contrast with previous studies ${ }^{27}$, our study could not show positive relationship between chronic urticaria and thyroid autoimmunity. This is likely to be because an insufficient number of patients were included or because TFT and thyroid autoantibodies were not routinely measured for all patients. Therefore, TFT alone is not enough to rule out thyroid disease and the thyroid antibody test should be carried out to rule out thyroid autoimmunity in CIU patients.

\section{CONCLUSION}

Patients with autoimmune and non-autoimmune chronic urticaria have no distinctive diagnostic clinical and histological features ${ }^{1}$. Hence, ASST is the only practicable test available to clinicians to detect autoimmune urticaria. The ASST test can be done by a dermatologist to determine whether chronic idiopathic urticaria is autoimmune in origin. This is especially important from a management viewpoint since immunosuppressive therapies may be tried if conventional approaches of management are unsuccessful.

\section{LIMITATION OF STUDY}

- The study was inherently limited by the small sample size and

- There was no any objective evidence of off antihistamine and oral steroids days during ASST test which may give false negative ASST results.

\section{REFERENCES}

1. Greaves M. Chronic urticaria. J Allergy Clin Immunol 2000;105:664-72.

2. Hide M, Francis DM, Grattan CE, Hakimi J, Kochan JP, Greaves MW. Autoantibodies against the high affinity IgE receptor as a cause of histamine release in chronic urticaria. $\mathrm{N}$ Engl J Med 1993;328:1599-604.

3. Godse KV. Autologous serum skin test in chronic idiopathic urticaria. Indian J Dermatol Venereol Leprol 2004;70:283-4

4. Fiebiger E, Maurer D, Holub H, Reininger B, Hartmann G, Woisetschlסger $M$, et al. Serum IgG autoantibodies directed against the alpha chain of Fc epsilon RI: A selective marker and pathogenetic factor for a distinct subset of chronic urticaria patients? J Clin Invest 1995:96:2606-12.

5. Ferrer M, Kinet JP, Kaplan AP. Comparative studies of functional and binding assays for IgG anti Fc epsilon Ria ( $\alpha$-subunit) in chronic urticaria. J Allergy Clin Immunol 1998;101:672-8.

6. Piconi S, Trabattoni D, lemoli E, Fusi ML, Villa ML, Milazzo F, et al. Immune profiles of patients with chronic idiopathic urticaria. Int Arch Allergy Immunol 2002;128:59-66.

7. Sabroe RA, Grattan CE, Francis DM, Barr RM, Kobza Black A, Greaves MW. The autologous serum skin test: A screening test for autoantibodies in chronic idiopathic urticaria. $\mathrm{Br} J$ Dermatol 1999;140:446-52.

8. Grattan CEH. Autoimmune urticaria. Immunol Allergy Clin N Am 2004;24:163-81.

9. Sabroe RA, Greaves MW. Chronic idiopathic urticaria with functional autoantibodies: 12 years on. $\mathrm{Br} J$ Dermatol 2006;154:813-19.

10. Sabroe RA, Seed PT, Francis DM, Barr RM, Kobza Black A, Greaves MW. Chronic idiopathic urticaria: Comparison of the clinical features of patients with and without anti- Fc epsilon RI or ani IgE antibodies. J Am Acad Dermatol 1999;40:443-50.

11. O'Donnell BF, Swana GT, Kobza Black A. Organ and nonorgan specific autoimmunity in chronic urticaria. $\mathrm{Br} J$ Dermatol 1995;133:42A.

12. Vohra S, Sharma NL, Mahajan VK. Autologous serum skin test: Methodology, interpretation and clinical applications. Indian J Dermatol Venereol Leprol 2009;75:545-8.

13. Kulthanan K, Jiamton S, Gorvanich T, Pinkaew S. Autologous Serum Skin Test in Chronic Idiopathic Urticaria: Prevalence, 
Correlation and Clinical Implications. Asian Pacific J Allergy Immunol 2006;24:201-6.

14. Kaplan AP. Chronic urticaria: Pathogenesis and treatment. J Allergy Clin Immunol 2004;114:465-74.

15. Grattan CEH. Autoimmune urticaria. Immunol Allergy Clin N Am 2004;24:163-81.

16. Sabroe RA, Fiebiger E, Francis DM, Maurer D, Seed PT, Grattan CE, et al. Classification of anti-FceRI and anti-IgE autoantibodies in chronic idiopathic urticaria and correlation with disease severity. J Allergy Clin Immunol 2002;110:492-9.

17. Toubi E, Kessel A, Avshovich N, Bamberger E, Sabo E, Nusem D, et al. Clinical and laboratory parameters in predicting chronic urticaria duration: A prospective study of 139 patients. Allergy 2004;59:869-73

18. O'Donnell BF, Francis DM, Swana GT, Seed PT, Black AK, Greaves MW. Thyroid autoimmunity in chronic urticaria. Br J Dermatol 2005;153:331-5.

19. Bakos N, Hillander M. Comparison of chronic autoimmune urticaria with chronic idiopathic urticaria. Int J Dermatol 2003;42:613-5.

20. Mamatha G, Balachandran C, Smitha P. Chronic idiopathic urticaria: Comparison of clinical features with positive autologous serum skin test. Indian J Dermatol Venereol Leprol 2008;74:105-8.

21. Nettis E, Dambra P, D'Oronzio L, Cavallo E, Loria MP, Fanelli M, et al. Reactivity to autologous serum skin test and clinical features in chronic idiopathic urticaria. Clin Exp Dermatol 2002;27:29-31.

22. Fusari A, Colangelo C, Bonifazi F, Antonicelli L. The autologous serum skin test in the follow-up of patients with chronic urticaria. Allergy 2005;60:256-8.

23. Godse K. Methotrexate in autoimmune urticaria. Indian J Dermatol Venereol Leprol 2004;70:377.

24. Baskan EB, Turker T, Gulten M, Sukran T. Lack of correlation between Helicobacter pylori infection and autologous serum skin test in chronic idiopathic urticaria. Int J Dermatol 2005;44:993-5.

25. Swerdt AD, Keybus CV, Kasran A, Cadot P, Neyens K, Coorevits L, et al. Detection of basophil-activating IgG autoantibodies in chronic idiopathic urticaria by induction of CD63. J Allergy Clin Immunol 2005;116:662-7.

26. Zweiman B, Valenzano M, Atkins PC, Tanus T, Getsy JA. Characteristics of histamine-releasing activity in the sera of patients with chronic idiopathic urticaria. J Allergy Clin Immunol 1996;98: 89-98.

27. Leznoff A, Sussman GL. Syndrome of idiopathic chronic urticaria and angioedema with thyroid autoimmunity. J Allergy Clin Immunol 1989;84:66-71. 